\title{
Loss to follow-up in anti-HCV-positive patients in a Brazilian regional outpatient clinic
}

\author{
L.C. Mendes ${ }^{1,2}$, S.M. Ralla ${ }^{2}$ and A.G. Vigani ${ }^{1,2}$ \\ ${ }^{1}$ Universidade Estadual de Campinas, Campinas, SP, Brasil \\ ${ }^{2}$ Ambulatório Municipal de Hepatites Virais, Campinas, SP, Brasil
}

\begin{abstract}
Loss to follow-up (LF), which refers to patients who started care but voluntary stopped it, is a problem for patients with chronic disease. We aimed to estimate the rate of LF among patients seropositive for hepatitis C virus (HCV) and identify possible demographic and lifestyle risk factors associated with LF. From January 2009 through December 2012, 1010 anti-HCV-positive patients were included in the study. Among participants, $223(22.1 \%)$ met the case definition for LF (more than 1-year elapsed since the last clinical appointment). Among 787 patients who remained in follow-up, $372(47.2 \%)$ were discharged after undetectable HCV RNA, 88 (11.1\%) were transferred (and remained on regular follow-up at the destination), and 25 (3.1\%) died. According to univariate analysis, male gender, absence of a life partner, black race, psychiatric illness, previous alcohol abuse, previous or current recreational drug use, and previous or current smoking were significantly associated with LF. In multivariate analysis, absence of a life partner (adjusted odds ratio $(A O R)=1.44 ; 95 \%$ confidence interval $(95 \% \mathrm{Cl})=1.03-2.02)$, black race $(A O R=1.81,95 \% \mathrm{Cl}=1.12-2.89)$, psychiatric illness $(A O R=1.77,95 \% \mathrm{Cl}=1.14-2.73)$, and the presence of at least one lifestyle risk factor (pertaining to substance abuse) $(A O R=1.95,95 \% \mathrm{Cl}=1.29-2.94)$ were independently associated with $L F$. Our study provides an estimate of the incidence of LF among anti-HCV-positive patients and identifies risk factors associated with this outcome. In addition, these results can help clinicians recognize patients at risk for LF, who require additional support for the continuity of care.
\end{abstract}

Key words: Hepatitis C; Follow-up management; Adherence

\section{Introduction}

Hepatitis $\mathrm{C}$ is the leading cause of liver disease worldwide and currently $20-30 \%$ of individuals with chronic hepatitis $\mathrm{C}$ develop cirrhosis. By 2030 this rate is expected to rise to $45 \%(1,2)$. Among patients with hepatitis $C$ and cirrhosis, the annual risk of hepatocellular carcinoma ( $\mathrm{HCC})$ is $3-5 \%$. With the new direct-acting agents against hepatitis $\mathrm{C}$ virus (HCV) infection, sustained virological response (SVR) is possible in over $80 \%$ of cases. SVR is associated with increased survival and a significant reduction in complications such as hepatocellular carcinoma and hepatic decompensation $(3,4)$. However, the successful treatment of patients with chronic hepatitis $C$ and prevention of further complications require regular medical follow-up.

Loss to follow-up (LF), which refers to patients who started care but voluntarily stopped it, is a problem for patients with chronic disease. $(5,6)$. There has not been any study in the HCV-infected population in Brazil specifically designed to assess LF rates and few are available worldwide, so the magnitude of this problem remains unknown. In addition, little is known about risk factors associated with LF in patients with $\mathrm{HCV}$ infection in outpatient settings.

A clear understanding of how patient characteristics influence LF can assist treatment programs to institute measures for improving patient adherence directed at those with increased risk for interruption of follow-up, such as intensified outreach or follow-up services.

We aimed to identify the rate of LF in outpatients positive for antibodies against HCV (anti-HCV), and demographic and lifestyle risk factors associated with LF.

\section{Material and Methods}

\section{Study population}

Anti-HCV-positive patients from a public regional outpatient clinic for viral hepatitis in the city of Campinas, São Paulo State, Southeast region of Brazil were included in this study. Patients enrolled in the study started care from January 2009 to December 2012. Samples were tested against HCV antibodies using Abbott AxSYM AntiHCV 3.0 (Abbott Laboratories, Germany). Quantitative 
serum HCV RNA was assessed by Amplicor HCV 2 (Roche Diagnostics Systems Inc., USA) upon entry into care in order to confirm chronic hepatitis $C$ infection.

\section{Data collection}

We collected patient data using standardized questionnaires indicated by healthcare providers filled during the first visit to the clinic. The questionnaire included demographics information and medical history with special emphasis on comorbidities and lifestyle. Lifestyle encompassed previous or current alcohol abuse, previous or current recreational drug use, and tobacco smoking. Alcohol abuse was defined as seven or more drinks per week. Current alcohol abuse, illicit drug use, and smoking were considered active, if it occurred during the six months prior to study enrollment, and previous, if discontinued before that period. In case of LF, a second questionnaire was used for collecting information regarding reasons for care interruption. All information collect was entered into EPI INFO 2000 (Centers for Disease Control and Prevention, USA).

\section{Definition of loss to follow-up}

We defined LF as patients with more than 1 year since last clinic appointment. When patients presented more than one interruption during the study period, only the first episode was considered for analysis. LF rate was calculated using the number of LF events divided by the total number of patients who started treatment during the study period. Patients transferred to other health facilities were not considered as LF.

When patients interrupted clinic visits for over 12 months, a recall was attempted through available contact information. We also reviewed medical records to locate information related to death, hospital transfers, and any other relevant data.

\section{Factors associated with LF}

We compared the characteristics of patients who interrupted care with patients who attended the clinic regularly during the study period to identify factors associated with follow-up interruption. Demographic data included age at enrollment, gender, and race. Lifestyle data included previous or current alcohol abuse, recreational drug use, and tobacco smoking.

\section{Statistical analyses}

Continuous variables are reported as means and standard deviations, and categorical variables as frequencies, unless otherwise stated. The $X^{2}$ test was used for nominal categorical variables. Analysis of variance (standard or nonparametric, as appropriate) was used to test associations between continuous and categorical variables. Variables for which an association was suspected in the univariate analysis (i.e., $P \leqslant 0.20$ ) were included in a stepwise logistic regression model. All analyses were performed with EPI INFO 2000. A significance level of $5 \%$ $(P<0.05)$ was used throughout.

\section{Ethical considerations}

This study is the result of a retrospective evaluation of medical records of patients treated at a municipal clinic under routine care, and it was approved by the Ethical Committee of the "Centro Infantil de Investigações Hematológicas Dr. Domingo A. Boldrini" (process \#39002414.5. 0000.5376). Patient informed consent was not deemed necessary for this study.

\section{Results}

Between March 1 and December 31, 2012, 1010 antiHCV-positive patients started care in this outpatient clinic. Patient characteristics are show in Table 1. Among all patients, $55.1 \%$ were male and the mean age at the beginning of follow-up was 49 years. Information about race was available for $987(97.7 \%)$ patients of which $11.1 \%$ were black. Almost $45 \%$ (444/992) of patients reported not having a life partner, such as spouse or domestic partner. In regards to comorbidities, information was available for 943 patients, of which 136 (14.4\%) reported diabetes and 40 (13.4\%), psychiatric disorders. Moreover, self-reported information on lifestyle risk factors was available for 979 patients with $657(67.1 \%)$ who had at least one lifestyle risk factor. The most common lifestyle risk factor was current or previous alcohol consumption $(n=495 ; 50.6 \%)$ and current tobacco smoking $(n=442$; $41.4 \%$ ). Among all enrolled patients, when LF occurred, $576(57.0 \%)$ had detectable serum HCV RNA, 372 (36.8\%) had undetectable serum HCV RNA, and the remaining $62(6.1 \%)$ patients were lost to follow-up before

Table 1. Characteristics of the 1010 anti-HCV-positive patients.

\begin{tabular}{lcc}
\hline Characteristic & Total $^{*}$ & $\mathrm{n}(\%)$ \\
\hline Male gender & 1010 & $557(55.1)$ \\
Age (years) (mean \pm SD) & 1010 & $48.3 \pm 12.47$ \\
Black race & 987 & $110(11.1)$ \\
Absence of a life partner & 992 & $444(44.8)$ \\
Presence of comorbidity & 943 & $545(57.8)$ \\
Diabetes & 943 & $136(14.4)$ \\
Psychiatric illness & 943 & $126(13.4)$ \\
Presence of $\geqslant 1$ lifestyle factor & 979 & $657(67.1)$ \\
Previous alcohol abuse & 979 & $495(50.6)$ \\
Current alcohol abuse & 979 & $266(22.0)$ \\
Previous illicit drug use & 979 & $369(30.6)$ \\
Current illicit drug use & 979 & $57(4.7)$ \\
Previous smoking & 979 & $442(41.4)$ \\
Current smoking & 979 & $299(24.8)$ \\
\hline
\end{tabular}

* Total number of patients for whom information regarding risk factors was available in medical records. 


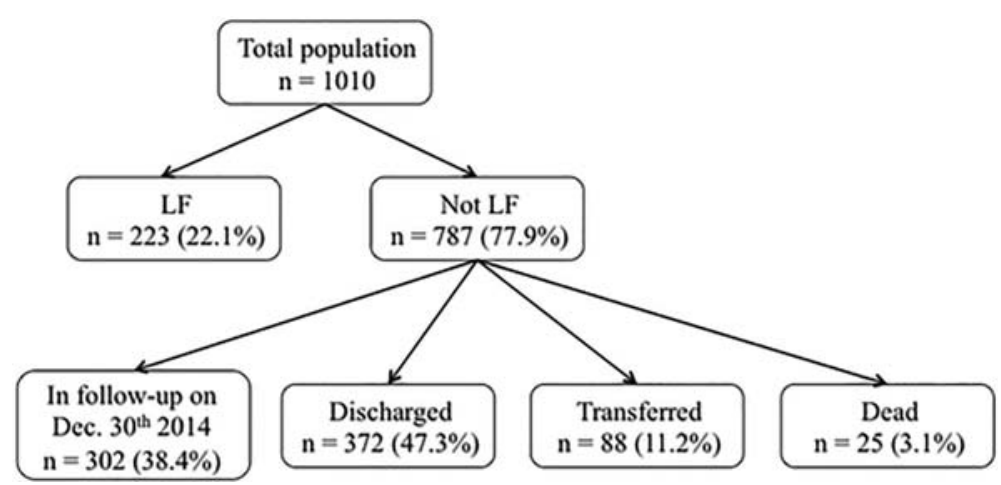

Figure 1. Outcomes of the study population. LF: lost to follow-up.

Table 2. Risk factors for loss to follow-up in univariate analysis.

\begin{tabular}{lcccl}
\hline Risk factor & Patients lost to follow-up & Patients in care & No. of patients* & P \\
\hline Age (years) (mean \pm SD) & $47.7 \pm 10.8$ & $48.5 \pm 12.9$ & 1010 & 0.3 \\
Male, n (\%) & $141(63.2)$ & $416(52.9)$ & 1010 & $\mathbf{0 . 0 0 5}$ \\
Absence of a life partner, n (\%) & $120(55.6)$ & $324(41.8)$ & 992 & $\mathbf{0 . 0 0 0 3}$ \\
Black race, n (\%) & $37(17.3)$ & $73(9.4)$ & 987 & $\mathbf{0 . 0 0 1}$ \\
Comorbidities, n (\%) & $107(56.9)$ & $438(58.0)$ & 943 & 0.7 \\
Diabetes, n (\%) & $20(10.6)$ & $116(15.4)$ & 943 & 0.09 \\
Psychiatric illness, n (\%) & $40(21.3)$ & $86(11.4)$ & 943 & $\mathbf{0 . 0 0 0 3}$ \\
Lifestyle risk factors, n (\%) & $163(79.9)$ & $494(63.7)$ & 979 & $\mathbf{0 . 0 0 0 0 1}$ \\
Previous alcohol abuse, n (\%) & $122(59.8)$ & $373(48.1)$ & 979 & $\mathbf{0 . 0 0 3}$ \\
Current alcohol abuse, n (\%) & $55(27.0)$ & $172(22.2)$ & 979 & 0.1 \\
Previous illicit drug use, n (\%) & $93(45.6)$ & $244(31.5)$ & 979 & $\mathbf{0 . 0 0 0 1}$ \\
Current illicit drug use, n (\%) & $18(8.8)$ & $37(4.4)$ & 979 & $\mathbf{0 . 0 2}$ \\
Previous smoking, n (\%) & $105(51.5)$ & $377(43.5)$ & 979 & $\mathbf{0 . 0 4}$ \\
Current smoking, n (\%) & $75(36.8)$ & $195(25.2)$ & 979 & $\mathbf{0 . 0 0 0 9}$ \\
\hline
\end{tabular}

* Total number of patients for whom information regarding risk factors was available in medical records.

RNA testing. Specific treatment for HCV had not yet been prescribed for any patient when LF occurred.

The outcomes of the study population are showed in Figure 1. Case definition for LF was met in $22.1 \%$ of patients (223 of 1010) and of these, 5 died after LF. Among the 1010 patients, 787 (77.9\%) did not meet the case definition of LF. Among these, $47.2 \%$ were discharged after undetectable levels of HCV RNA, 88 of 787 $(11.2 \%)$ were transferred to other facilities, and $25(3.1 \%)$ died. The median duration of follow-up for lost patients was 4 months and $37.7 \%$ of those patients interrupted care with less than 30 days after starting it.

According to univariate analysis, gender, absence of a life partner, black race, psychiatric illness, and at least one lifestyle risk factor were significantly associated with LF (Table 2). Single black male patients were more likely to experience LF. Among lifestyle factors, previous alcohol abuse, previous and current illicit drug use, and previous or current tobacco smoking were associated with LF.
Table 3. Risk factors for loss to follow-up in multivariate analysis.

\begin{tabular}{lcl}
\hline Risk factor & Adjusted OR $(95 \% \mathrm{Cl})$ & $\mathrm{P}$ \\
\hline Male & $1.16(0.81-1.65)$ & 0.41 \\
Absence of life partner & $1.44(1.03-2.02)$ & $\mathbf{0 . 0 2}$ \\
Black race & $1.80(1.12-2.89)$ & $\mathbf{0 . 0 1}$ \\
Psychiatric illness & $1.77(1.14-2.73)$ & $\mathbf{0 . 0 0 9}$ \\
Diabetes & $0.71(0.4-1.21)$ & 0.21 \\
Any lifestyle risk factor & $1.95(1.29-2.94)$ & $\mathbf{0 . 0 0 1}$ \\
\hline
\end{tabular}

* Lifestyle risk factors included previous or current alcohol abuse, recreational drug use, and smoking.

In the multivariate analysis, absence of a life partner, black race, psychiatric illness and substance abuse were independently associated with LF (Table 3).

We were able to locate $104(46.6 \%)$ of the 223 patients lost to follow-up and only $33(14.8 \%)$ returned to follow-up 
Table 4. Reasons for loss to follow-up among 223 patients.

\begin{tabular}{lr}
\hline & $\mathrm{n}(\%)$ \\
\hline Conflicts with work schedule & $20(9.0)$ \\
Incarceration & $17(7.6)$ \\
City relocation & $15(6.7)$ \\
Absence of symptoms & $10(4.5)$ \\
Recreational drugs abuse & $9(4.0)$ \\
Social condition & $8(3.6)$ \\
Alcohol abuse & $7(3.1)$ \\
Psychiatric disease & $4(1.8)$ \\
Others & $11(4.9)$ \\
Unknown & $122(54.7)$ \\
\hline
\end{tabular}

visits. The most frequent reason for LF stated by patients was incompatibility between work hours and scheduled clinical appointments, followed by incarceration (Table 4). However, for $122(54.7 \%)$ patients no reason for losing follow-up could be provided.

\section{Discussion}

With recent advances in anti HCV treatment, SVR can be reached in $80-90 \%$ of patients (7). In this new scenario, it is now possible to envision a drastic reduction of $\mathrm{HCV}$ prevalence, but major challenges remain. One of them, according to our findings, is LF. Among anti-HCVpositive patients, we found a LF rate of $22.1 \%$, and male, absence of a partner, black race and the presence of one lifestyle risk factor were independently associated with LF. In addition, almost $40 \%$ of patients were lost to follow-up within less than one month after starting care. Finally, less than $15 \%$ of patients lost to follow-up returned to the clinic after being actively recalled.

There have been few studies evaluating LF and non-adherence to care in the HCV infected population. Notably, most data regarding non-adherence and patient compliance are related to treatment-associated factors, and are not regarded as a primary outcome (8-11). For this reason, many patients who quit follow-up before complete staging and treatment indication are not included in those studies. Therefore, evaluating patient adherence to care in the initial steps of HCV evaluation is paramount for a more complete understanding of the problem.

Before therapy has been indicated, treatment eligibility is directly influenced by patient compliance with follow-up. In Brazil, eligibility for treatment has been evaluated in $\mathrm{HIV} / \mathrm{HCV}$ co-infected patients and non-adherence to therapy was found to be responsible for $31.4 \%$ of ineligibility for HCV treatment (12). According to our findings, patients are lost to follow-up soon after initiating care, which has also been observed in previous studies $(13,14)$. A Greek study found that $27 \%$ of anti-HCV-positive were lost to follow-up before HCV RNA testing, and the majority of patients who did not receive treatment were lost to follow-up (15). When evaluating LF as a risk factor for treatment discontinuation and, similarly, for treatment failure, observational studies have found that nonadherence was directly associated with non-response to therapy in 18 to $29 \%$ of patients $(8,9,16-18)$. Our results corroborate those findings and call attention to LF as an important reason for treatment ineligibility.

Non-adherence to care is a known issue for chronic illnesses. In terms of chronic, mostly asymptomatic infections, HIV and HCV share some similarities, especially considering the need for close monitoring and follow-up. In our cohort, the LF was similar to that observed around the world among HIV-positive patients. Among HIV-positive patients, the LF rates range from 13.4 to $25 \%(13,19-21)$. In Brazil, among HIV-positive patients, the LF rates ranged from 17.6 to $23.9 \%(22,23)$. The great variability observed in HIV-positive populations can be related to the lack of standardized definitions on follow-up time, which ranges from 3 months in some studies to up to 12 months in others (22,24-27). Secondly, the different population characteristics in the various regions of the world that could impact patients' ability to consistently maintain access to health care (28), can influence LF rates. It is necessary to consider that these differences may also be true for HCV infection.

Establishing risk factors associated with LF has the potential to identify patients that require additional efforts to remain in follow-up. Similar to our findings, previous studies have also found a correlation between both alcohol consumption and psychiatric illness with ineligibility for treatment and also with treatment failures $(10,12,29)$. The association found between absence of a partner and LF is difficult to be fully understood. A possible explanation is that patients without partners do not possess sufficient emotional and social support systems. Finally, a chronic physical disease not only has direct consequences for the patient, but can also disrupt the normal life of the healthy partner (30).

According to the NHANES report, the HCV prevalence is $14 \%$ among black people and $1.6 \%$ in the general population in the USA, and black people with chronic hepatitis $C$ have higher age-adjusted mortality rates from cirrhosis and $\mathrm{CHC}$ than non-Hispanic whites with chronic hepatitis $C$ (31). According to our findings, black race was a recognizable risk factor for LF. It is possible that the high rate of black patients who are lost to follow-up prior to fibrosis staging and treatment indication may be responsible for some of the high $\mathrm{CHC}$ incidence in this group, and they might not be represented in studies of treatment response.

In patients with hepatitis $C$, the association between psychiatric disorders and LF is common. Patients infected with $\mathrm{HCV}$ have a high prevalence $(50 \%)$ of psychiatric disease. In addition, the lifetime prevalence of psychosis, 
anxiety, substance abuse, and personality disorders are all higher among patients with HCV than the general USA population (32). The association between lifestyle factors (excess alcohol consumption, tobacco smoking or recreational drug use) could also be associated with impulsive personality and hopelessness (33). In that sense, depression is the most commonly diagnosed mood disorder in HCV-infected patients and has been extensively associated with barriers to compliance with care and treatment adherence (12,34-36).

We were able to locate only $46.6 \%$ of the patients lost to follow-up. This finding suggests that efforts should be made to encourage patients to provide as many personal contacts as possible, and additional information on family members or friends. In addition, for $54.7 \%$ of LF patients no reason for non-compliance with care could be found. This demonstrates that LF is a complex multifactorial process and understanding patient-associated reasons for non-compliance can present a challenge. Based on our findings, we suggest that recalling patients is necessary but not sufficient to ensure continuity of care. Similar

\section{References}

1. Davis GL, Albright JE, Cook SF, Rosenberg DM. Projecting future complications of chronic hepatitis $C$ in the United States. Liver Transpl 2003; 9: 331-338, doi: 10.1053/ jlts.2003.50073.

2. Armstrong GL, Alter MJ, McQuillan GM, Margolis HS. The past incidence of hepatitis $C$ virus infection: implications for the future burden of chronic liver disease in the United States. Hepatology 2000; 31: 777-782, doi: 10.1002/ hep. 510310332.

3. Cardoso AC, Moucari R, Figueiredo-Mendes C, Ripault MP, Giuily N, Castelnau C, et al. Impact of peginterferon and ribavirin therapy on hepatocellular carcinoma: incidence and survival in hepatitis $C$ patients with advanced fibrosis. J Hepatol 2010; 52: 652-657, doi: 10.1016/j.jhep.2009. 12.028.

4. Deuffic-Burban S, Babany G, Lonjon-Domanec I, Deltenre P, Canva-Delcambre V, Dharancy S, et al. Impact of pegylated interferon and ribavirin on morbidity and mortality in patients with chronic hepatitis $C$ and normal aminotransferases in France. Hepatology 2009; 50: 1351-1359, doi: 10.1002/ hep.23159.

5. Rosen S, Fox MP. Retention in HIV care between testing and treatment in sub-Saharan Africa: a systematic review. PLoS Med 2011; 8: e1001056, doi: 10.1371/journal. pmed.1001056.

6. Mugusi F, Josiah R, Moshi A, Chale S, Bakari M, Aris E, et al. Dropouts in a long-term follow-up study involving voluntary counseling and HIV testing: experience from a cohort of police officers in Dar Es Salaam, Tanzania. J Acquir Immune Defic Syndr 2002; 30: 119-123, doi: 10.1097/00126334-200205010-00016.

7. Ghany MG, Liang TJ. Current and future therapies for hepatitis C virus infection. N Engl J Med 2013; 369: 679680, doi: 10.1056/NEJMc1307589. studies in the HIV-infected population showed that 4 to $28 \%$ of patients returned to care after recall $(37,38)$.

Our study has some limitations. We were not able to examine social characteristics, such as homelessness and unemployment states because these variables were not systematically reported in the medical records. Finally, data on telephone number and name of primary care physician were retrospectively collected after reviewing patient's charts, and might lack precision.

In conclusion, our study provides an estimated incidence of LF among anti-HCV-positive patients and identifies risk factors associated with this outcome. Importantly, our results comprise LF data from patients at the first stage in the HCV chain of care. In addition, our study can help clinicians recognize patients at risk for non-compliance and who require additional support for retention of care. Longitudinal studies are urgently needed to better identify these patients as well as variables that affect their retention in care. A clear understanding of how patient characteristics influence treatment adherence can assist the development of better strategies to improve patient outcomes.

8. Tanioka D, Iwasaki $\mathrm{Y}$, Araki $\mathrm{Y}$, Osawa T, Ikeda $\mathrm{H}$, Ando $\mathrm{M}$, et al. Factors associated with adherence to combination therapy of interferon and ribavirin for patients with chronic hepatitis C: importance of patient's motivation and physician's treatment experience. Liver Int 2009; 29: 721-729, doi: 10.1111/j.1478-3231.2008.01964.x.

9. Raptopoulou M, Tsantoulas D, Vafiadi I, Ketikoglou I, Paraskevas E, Vassiliadis T, et al. The effect of adherence to therapy on sustained response in daily or three times a week interferon alpha- $2 \mathrm{~b}$ plus ribavirin treatment of naive and nonresponder chronic hepatitis $C$ patients. $J$ Viral Hepat 2005; 12: 91-95, doi: 10.1111/j.1365-2893.2005.00549.x.

10. Castera L, Constant A, Henry $C$, Champbenoit $P$, Bernard $\mathrm{PH}$, de Ledinghen $\mathrm{V}$, et al. Impact on adherence and sustained virological response of psychiatric side effects during peginterferon and ribavirin therapy for chronic hepatitis C. Aliment Pharmacol Ther 2006; 24: 1223-1230, doi: 10.1111/j.1365-2036.2006.03107.x.

11. Cheng SJ, Bonis PA, Lau J, Pham NQ, Wong JB. Interferon and ribavirin for patients with chronic hepatitis $C$ who did not respond to previous interferon therapy: a meta-analysis of controlled and uncontrolled trials. Hepatology 2001; 33: 231-240, doi: 10.1053/ihep.2001.20675.

12. Mendes-Correa MC, Martins LG, Tenore S, Leite OH, Leite $A G$, Cavalcante AJ, et al. Barriers to treatment of hepatitis $C$ in HIV/HCV coinfected adults in Brazil. Braz $\mathrm{J}$ Infect Dis 2010; 14: 237-241, doi: 10.1016/S1413-8670(10)70050-7.

13. Ndiaye B, Ould-Kaci K, Salleron J, Bataille P, Bonnevie F, Choisy $\mathrm{P}$, et al. Incidence rate and risk factors for loss to follow-up in HIV-infected patients from five French clinical centres in Northern France - January 1997 to December 2006. Antivir Ther 2009; 14: 567-575.

14. Arici C, Ripamonti D, Maggiolo F, Rizzi M, Finazzi MG, Pezzotti $P$, et al. Factors associated with the failure of 
HIV-positive persons to return for scheduled medical visits. HIV Clin Trials 2002; 3: 52-57, doi: 10.1310/2XAK-VBT89NU9-6VAK.

15. Papadopoulos N, Manolakopoulos S, Deutsch M, Mela M, Christidou A, Katoglou A, et al. Frequency and predictors of no treatment in anti-hepatitis $C$ virus-positive patients at tertiary liver centers in Greece. Eur J Gastroenterol Hepatol 2013; 25: 587-593, doi: 10.1097/MEG.0b013e32835cb5c0.

16. McHutchison JG, Manns M, Patel K, Poynard T, Lindsay KL, Trepo C, et al. Adherence to combination therapy enhances sustained response in genotype-1-infected patients with chronic hepatitis C. Gastroenterology 2002; 123: 10611069, doi: 10.1053/gast.2002.35950.

17. Kemmer N, Neff GW. Managing chronic hepatitis $C$ in the difficult-to-treat patient. Liver Int 2007; 27: 1297-1310, doi: 10.1111/j.1478-3231.2007.01613.x.

18. Morisco F, Stroffolini T, Medda E, Amoruso DC, Almasio PL, Villa $E$, et al. Retrospective, observational, multicentre study on an Italian population affected by chronic hepatitis $C$ who failed to clear HCV-RNA after the combined therapy (PEGIFN and ribavirin): NADIR study. J Viral Hepat 2010; 17: 427-434, doi: 10.1111/j.1365-2893.2009.01200.x.

19. Schepens T, Morreel S, Florence E, Koole O, Colebunders $R$. Incidence and risk factors associated with lost to followup in a Belgian cohort of HIV-infected patients treated with highly active antiretroviral therapy. Int J STD AIDS 2010; 21: 765-769, doi: 10.1258/ijsa.2010.010303.

20. Fleishman JA, Yehia BR, Moore RD, Korthuis PT, Gebo KA. Establishment, retention, and loss to follow-up in outpatient HIV care. J Acquir Immune Defic Syndr 2012; 60: 249-259, doi: 10.1097/QAl.0b013e318258c696.

21. Rice BD, Delpech VC, Chadborn TR, Elford J. Loss to follow-up among adults attending human immunodeficiency virus services in England, Wales, and Northern Ireland. Sex Transm Dis 2011; 38: 685-690, doi: 10.1097/ OLQ.0b013e318214b92e.

22. de Almeida MC, de Jesus PN, do Socorro Lina van Keulen, Jacome GP, Fernandes GC, Yokoo EM, et al. Loss to followup in a cohort of HIV-infected patients in a regional referral outpatient clinic in Brazil. AIDS Behav 2014; 18: 2387-2396, doi: 10.1007/s10461-014-0812-1.

23. Pacheco AG, Saraceni V, Tuboi SH, Moulton LH, Chaisson $\mathrm{RE}$, Cavalcante SC, et al. Validation of a hierarchical deterministic record-linkage algorithm using data from 2 different cohorts of human immunodeficiency virus-infected persons and mortality databases in Brazil. Am J Epidemiol 2008; 168: 1326-1332, doi: 10.1093/aje/kwn249.

24. Lanoy E, Lewden C, Lievre L, Tattevin P, Boileau J, Aouba A, et al. How does loss to follow-up influence cohort findings on HIV infection? A joint analysis of the French hospital database on HIV, Mortalite 2000 survey and death certificates. HIV Med 2009; 10: 236-245, doi: 10.1111/j.1468-1293.2008.00678.x.

25. Dalal RP, MacPhail C, Mqhayi M, Wing J, Feldman C, Chersich MF, et al. Characteristics and outcomes of adult patients lost to follow-up at an antiretroviral treatment clinic in johannesburg, South Africa. J Acquir Immune Defic Syndr 2008; 47: 101-107, doi: 10.1097/QAI.0b013e31815b833a.

26. Mocroft A, Kirk O, Aldins P, Chies A, Blaxhult A, Chentsova $\mathrm{N}$, et al. Loss to follow-up in an international, multicentre observational study. HIV Med 2008; 9: 261-269, doi: 10.1111/j.1468-1293.2008.00557.x.

27. Palombi L, Marazzi MC, Guidotti G, Germano P, Buonomo $E$, Scarcella $P$, et al. Incidence and predictors of death, retention, and switch to second-line regimens in antiretroviral-treated patients in sub-Saharan African Sites with comprehensive monitoring availability. Clin Infect Dis 2009; 48: 115-122, doi: 10.1086/593312.

28. Fraser HS, Allen C, Bailey C, Douglas G, Shin S, Blaya J. Information systems for patient follow-up and chronic management of HIV and tuberculosis: a life-saving technology in resource-poor areas. J Med Internet Res 2007; 9: e29, doi: 10.2196/jmir.9.4.e29.

29. Zayed N, Gamal EH, Elmakhzangy H, Seif M, El-Akel W, Awad T, et al. Therapeutic outcome of 6198 interferonnaive Egyptian patients with chronic hepatitis $\mathrm{C}$ : a reallife experience and lessons to be learned in DAAs' era. $J$ Viral Hepat (epub ahead of print) 2016, doi: 10.1111/ jvh.12514.

30. Baanders AN, Heijmans MJ. The impact of chronic diseases: the partner's perspective. Fam Community Health 2007; 30: 305-317, doi: 10.1097/01.FCH.0000290543.48576.cf.

31. Ward JW, Lok AS, Thomas DL, El-Serag HB, Kim WR. Report on a single-topic conference on "Chronic viral hepatitis - strategies to improve effectiveness of screening and treatment". Hepatology 2012; 55: 307-315, doi: 10.1002/hep.24797.

32. Rifai MA, Gleason OC, Sabouni D. Psychiatric care of the patient with hepatitis C: a review of the literature. Prim Care Companion J Clin Psychiatry 2010; 12, doi: 10.4088/ PCC.09r00877whi.

33. Mackinnon SP, Kehayes IL, Clark R, Sherry SB, Stewart SH. Testing the four-factor model of personality vulnerability to alcohol misuse: a three-wave, one-year longitudinal study. Psychol Addict Behav 2014; 28: 1000-1012, doi: 10.1037/ a0037244.

34. Bini EJ, Brau N, Currie S, Shen H, Anand BS, Hu KQ, et al. Prospective multicenter study of eligibility for antiviral therapy among 4,084 U.S. veterans with chronic hepatitis C virus infection. Am J Gastroenterol 2005; 100: 17721779, doi: 10.1111/j.1572-0241.2005.41860.x.

35. Fireman M, Indest DW, Blackwell A, Whitehead AJ, Hauser P. Addressing tri-morbidity (hepatitis C, psychiatric disorders, and substance use): the importance of routine mental health screening as a component of a comanagement model of care. Clin Infect Dis 2005; 40 (Suppl 5): S286S291, doi: $10.1086 / 427442$.

36. Nguyen HA, Miller Al, Dieperink E, Willenbring ML, Tetrick LL, Durfee JM, et al. Spectrum of disease in U.S. veteran patients with hepatitis C. Am J Gastroenterol 2002; 97: 1813-1820, doi: 10.1111/j.1572-0241.2002.05800.x.

37. Stringer JS, Zulu I, Levy J, Stringer EM, Mwango A, Chi BH, et al. Rapid scale-up of antiretroviral therapy at primary care sites in Zambia: feasibility and early outcomes. JAMA 2006; 296: 782-793, doi: 10.1001/jama.296.7.782.

38. Catz SL, McClure JB, Jones GN, Brantley PJ. Predictors of outpatient medical appointment attendance among persons with HIV. AIDS Care 1999; 11: 361-373, doi: 10.1080/ 09540129947983. 